\title{
CARACTERIZACIÓN Y OPTIMIZACIÓN DE UNA SAUNA SOLAR SECO CON ACUMULADORES TÉRMICOS*
}

\author{
CHARACTERIZATION AND OPTIMIZATION OF A SOLAR DRY SAUNA \\ WITH THERMAL STORAGE
}

${ }^{1}$ Carlos Armando Polo Bravo; ${ }^{2}$ Gohnny Acero Laura

\begin{abstract}
RESUMEN
El presente trabajo experimental tiene como objetivo principal caracterizar y optimizar térmica y energéticamente una Sauna Solar Seco (SSS) usando tres tipos de acumuladores térmicos; agua, piedras sin pintar, y piedras pintadas de negro mate, considerando el alto potencial solar de la región Tacna y ante la necesidad social de contar con sistemas que usen como fuente energética la energia solar para aplicaciones terapéuticas, que utilice energía limpia, renovable y de menor impacto ambiental. La caracterización térmica y energética experimental nos indican que la temperatura máxima alcanzada en el interior de caja absolvedora es de $77,46^{\circ} \mathrm{C}$, con humedad relativa mínima de $1,25 \%$, bajo una irradiancia solar de $838,1 \mathrm{~W} / \mathrm{m}^{2}$; con una longitud de onda que oscila entre 9,8 $\mu \mathrm{m}$ a $7,97 \mu \mathrm{m}$ de la radiación térmica emitida por la caja absolvedora, concordante con lo establecido por la Comisión Internacional de Iluminación (CIE), y una potencia emisiva que oscila entre 423,91 W/m a 991,24W/m, durante las 8:00 a las 20:00 horas, este resultado muestra la viabilidad de integración entre el sauna solar seco y la cabina de radiación infra-roja. La eficiencia media diaria del SSS con acumuladores térmicos de piedra pintada de negro mate, piedra sin pintar, agua contenida en botellas descartables y sin acumulador térmico, corresponde a 40,7\%,38,1\%,41,4\% y 38,7\%, respectivamente, lo que indica que el funcionamiento del sistema se puede optimizar usando acumuladores de piedra pintadas de negro mate, ya que bajo este escenario la eficiencia de la SSS es del $41,4 \%$, alcanzando temperatura internas de $94^{\circ} \mathrm{C}$.
\end{abstract}

Palabras Claves: sauna solar seco, acumuladores térmicos, energia solar.

\section{ABSTRACT}

This experimental work aims to characterize and optimize main thermal and energy one Solar Dry Sauna (SSS) using three types of thermal storage, water, stones unpainted and painted matte black stones, considering the high solar potential Tacna region, and to the social need of having such systems using solar energy source for therapeutic applications, which use clean, renewable energy. and lower environmental impact. Thermal characterization and experimental energy, indicate that the maximum temperature reached inside absolvedora box is $77,46^{\circ} \mathrm{C}$ with a minimum relative humidity of $1,25 \%$ under an irradiance of $838,1 \mathrm{~W} / \mathrm{m}^{2}$, with a wavelength ranging from 9,8 microns to 7,97 microns of thermal radiation emitted by the box absolvedora, consistent with the provisions of the International Commission on Illumination (CIE), and an emissive power of between $423,91 \mathrm{~W} / \mathrm{m}^{2}$ to $991,24 \mathrm{~W} / \mathrm{m}^{3}$ during the 8:00 to 20:00 pm, this result shows the feasibility of integrating the sun dry sauna cabin infrared radiation. The daily average efficiency of thermal storage SSS painted flat black stone, stone unpainted water in disposable bottles without heat storage corresponds to $40,7 \% ; 38,1 \% ; 41,4 \%$ and $38,7 \%$, respectively, indicating that the system operation can be optimized using accumulators matte painted stone denied because under this scenario SSS efficiency is $41,4 \%$ to internal temperature of $94^{\circ} \mathrm{C}$.

Key words: solar dry sauna, thermal storage, solar energy

\section{INTRODUCCIÓN}

La energía es considerada como el eje principal de todas las actividades que realizan los seres vivos $y$, por tanto, su incorrecta utilización es un aspecto muy preocupante en la actualidad, especialmente aquellas energias convencionales provenientes de combustibles fósiles, que han estado en el planeta desde hace millones de años, pero que hoy en día las reservas van disminuyendo con el pasar del tiempo, y que además, su consumo está generando grandes cantidades de gases tóxicos a la atmósfera causando varios problemas en el planeta como la lluvia ácida, "efecto invernadero", cambios bruscos en el clima, entre otros, produciendo en si elllamado calentamiento global.

De acuerdo con estos efectos tan alarmantes, se ha tomado en consideración, el aprovechamiento de las energías renovables, ya que estas se encuentran en gran cantidad, y poseen un gran potencial. Además, en nuestro país existe un alto déficit de recursos energéticos convencionales, por tanto, se pretende emplear al máximo

Magister Scientiac en Gestión Ambiental y Desarrollo Sostenible, Licenciado en Fisica. Centro de Energias Renovables de Tacna de la Universidad Nacional Jorge Basadre Grohmarn. Tacna-Perí Licenciado en Fisica A plicada. Centro de Energias Renovables de Tacna de la Universidad Nacional Jorge Basadre Grohmann. Tacna-Peri.

- Ponencia del XX Simposio Peruano de Energia Solar, 2013. Taena-Peni 
las fuentes energéticas renovables, no contaminantes; en este contexto el objetivo principal de este trabajo consiste en construir y optimizar un sistema alternativo al sauna convencional para aplicaciones terapéuticas, que utilice la energía solar como fuente energética.

El sistema fue diseñado de forma que la irradiancia solar global sea absorbida en un recinto aislado térmicamente, fenómeno conocido como efecto invernadero, en cuyo interior se encuentra instalada una caja absolvedora metálica pintada de negro, en la cual las personas pueden tomar su "baño" tipo sauna seco.

\subsection{Radiación solar}

El sol se comporta generalmente como un cuerpo negro emisor con una temperatura superficial efectiva de unos $5800 \mathrm{~K}$, la incidencia promedio fuera de la atmósfera terrestre es de $1353 \mathrm{~W} / \mathrm{m}^{2}$, llamada constante solar, el $95 \%$ de la cual es de longitud de onda inferior a $2 \mu \mathrm{m}$, gran parte de esta radiación es devuelta por reflexión al espacio, una gran proporción es absorbida selectivamente por diversos gases en la atmósfera, y otra es dispersada al espacio; de tal modo que después de un simple tránsito vertical a través de la capa atmosférica, aproximadamente $1000 \mathrm{~W} / \mathrm{m}^{2}$ en valor máximo, alcanza la superficie terrestre; esta radiación está formada aproximadamente en un $47 \%$ por el espectro visible, $46 \%$ por el espectro infrarrojo, y un $7 \%$ por el espectro ultravioleta. El espectro ultravioleta está determinado por longitudes de onda dentro del intervalo $(0,009-0,39) \mu \mathrm{m}$, el espectro visible por el intervalo $(0,39-$ $0,79) \mu \mathrm{m}$ y el infrarrojo por $(0,79-1000) \mu \mathrm{m}$. Figura $\mathrm{N}^{\circ} 01$, (Chassériaux J.M., 1990).

\subsection{Sauna solar seco}

La sauna solar es un sistema cuasi cerrado, parcialmente aislado térmicamente, que calienta el aire del ambiente interno por absorción de la irradiancia solar global incidente, está compuesto por el colector de la sauna solar, el cual es un subsistema de la sauna solar, recibe y absorbe la irradiancia solar global, emitiendo radiación infrarroja la cual es absorbida por aire interno, calentándolo progresivamente, bajo el mecanismo conocido como efecto invernadero. La cubierta transparente es el vidrio colocado

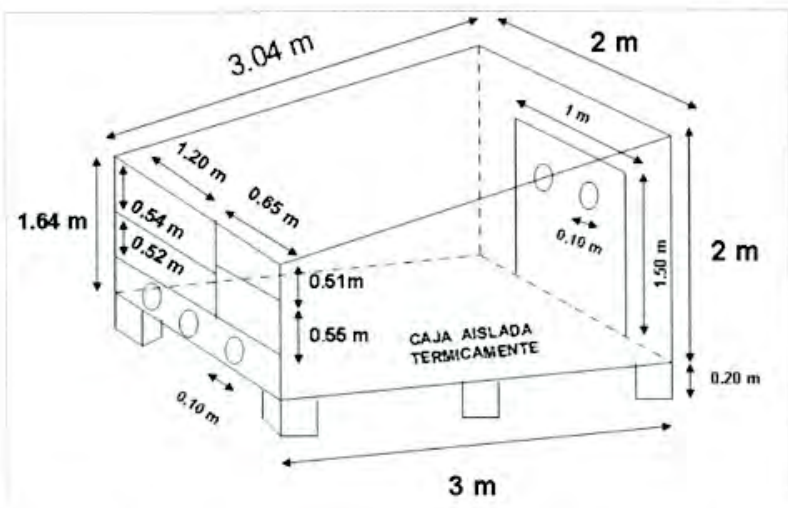

en la parte superior (cobertor) de la sauna solar, permite el paso de la radiación solar global y evita las pérdidas de calor por radiación y convección, con inclinación de $7^{\circ}$; la superficies absorbentes están constituidas por la caja metálica colocada en el interior de la caja aislada térmicamente, así como las paredes laterales internas pintadas de negro, el piso y el sistema de acumulación de calor, Figura $\mathrm{N}^{\circ} 02$ (Ordóñez M., 2004).

\subsection{Sauna finlandesa $o$ fauna seca}

Es un elemento terapéutico de relajación y bienestar físico-psíquico, estos beneficios se consiguen como consecuencia del clima creado en el interior, con temperaturas elevadas $(80-100){ }^{\circ} \mathrm{C}$ y niveles de humedad bajos $(10-20) \%$, según la Sociedad Internacional de Sauna (Acero Laura, G. 2011).

En la Figura $\mathrm{N}^{\circ} 02$, izquierda, se indican las dimensiones de la SSS, la base es de $3 \mathrm{~m} \times 2 \mathrm{~m}$, el techo $3,04 \mathrm{~m}$ $\times 2 \mathrm{~m}$, con una inclinación de $7^{\circ}$, la cara frontal $2 \mathrm{~m} \times 1,64 \mathrm{~m}$,

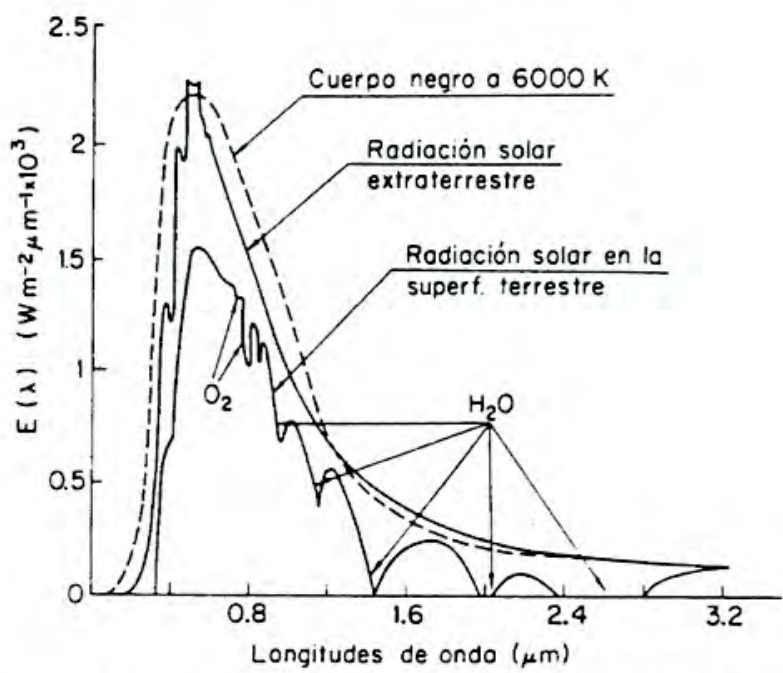

Figura $\mathrm{N}^{\circ} 01$. Espectro de la irradiancia solar global incidente sobre la superficie terrestre, la emitida por un cuerpo negro y la incidente sobre la capa exterior de la superficie terrestre (izquierda)

Fuente: Chassćriaux J.M., (1990)

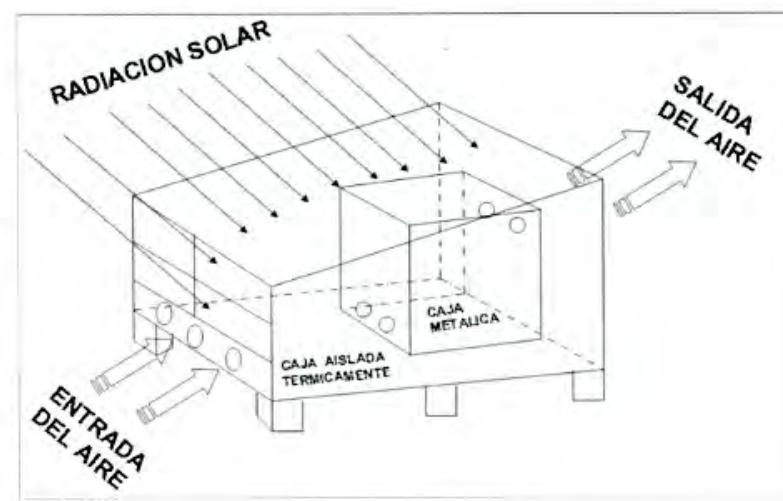

Figura $\mathrm{N}^{\circ} 02$. Dimensiones de la SSS, izquierda; Esquema general de la SSS, indicando sus partes principales, derecha 
cara posterior $2 \mathrm{~m} \times 2 \mathrm{~m}$, el volumen total del SS es de 13,08 $\mathrm{m} 3$, el sistema se ubica sobre el piso a una altura de $20 \mathrm{~cm}$.

En la Figura $\mathrm{N}^{\circ} 03$, derecha se muestra una vista general de la SSS totalmente construida, distinguiéndose las partes coberteras transparentes del techo, y parte de las paredes laterales y frontal, esta última orientada hacia el norte; las partes pintadas de blanco son paredes aisladas térmicamente, al igual que el fondo y la parte posterior.

\subsection{Balance energético}

El balance energético nos permite encontrar la distribución y transformación de la energía solar incidente sobre la SSS, en energía útil, energía almacenada, y las pérdidas térmicas. Para un intervalo de tiempo $d t$, el principio de conservación de la energía aplicado a una sauna, establece que:

$$
\begin{aligned}
& \left\{\begin{array}{l}
\text { Energia } \\
\text { Incidente }
\end{array}\right\}=\left\{\begin{array}{l}
\text { Energia } \\
\text { util }
\end{array}\right\}+\left\{\begin{array}{l}
\text { Energia } \\
\text { almacenada }
\end{array}\right\}+\left\{\begin{array}{l}
\text { Energia } \\
\text { perdida }
\end{array}\right\} \\
& Q_{a b s} d t=Q_{u} d t+Q_{p} d t+d E \\
& Q_{a b s}=Q_{u}+Q_{p}+\frac{d E}{d t}
\end{aligned}
$$

Dónde: Energía incidente es la energía solar incidente $\left(\mathrm{Q}_{\mathrm{bas}}\right)$ una vez que se ha trasmitido por el vidrio cobertor del techo, y parte de las paredes laterales y frontal; Energía útil es la energía calorífica que absorbe el aire interno del sistema $\left(Q_{\downarrow}\right)$; Energía almacenada es la energía absorbida por la caja metálica, por las paredes laterales, el piso del sistema y por los acumuladores térmicos como calor sensible (dE); Energía perdida es la energía calorífica perdida
Bravo C. y Acero G., Caracterización y Optimización de una sauna solar seco con acumuladores térmicos

por conducción, convección y radiación $\left(Q_{\mathrm{p}}\right)$. (Acero Laura, 2012)

Asumimos que la SSS funciona en régimen cuasi estacionario, por lo tanto la energía útil en la SSS es igual a:

$Q_{\mathrm{u}}=\mathrm{Q}_{\mathrm{abs}}-\mathrm{Q}_{\mathrm{p}}-\mathrm{dE} / \mathrm{dt}$

Esta ecuación nos permite determinar la eficiencia instantánea de la SSS, definida como:

$\mathbf{h}=$ Energía útil $/$ Energía incidente $\left.=Q_{u} / Q_{a b s}\right)$

Luego, la ecuación siguiente permite encontrar la eficiencia promedio diaria de la SSS, durante un periodo

$$
\tau=\sum_{i} \Delta t_{i}:
$$

$$
\eta_{d}=\frac{\sum_{i} Q_{u, i} \cdot \Delta t_{i}}{A \sum_{i} G_{i} \cdot \Delta t_{i}}
$$

Considerando lo indicado en el balance energético, que el medio que gana calor útil es el aire interno y la caja metálica, que el calor se almacena en todas las partes internas de la SSS y en los acumuladores térmicos usados como calor sensible y que las pérdidas térmicas se dan desde el interior al exterior del sistema por procesos combinados de conducción (paredes, fondo); convección (aire internoparedes internas; paredes externas-medio externo); radiación entre paredes laterales, fondo - medio exterior,

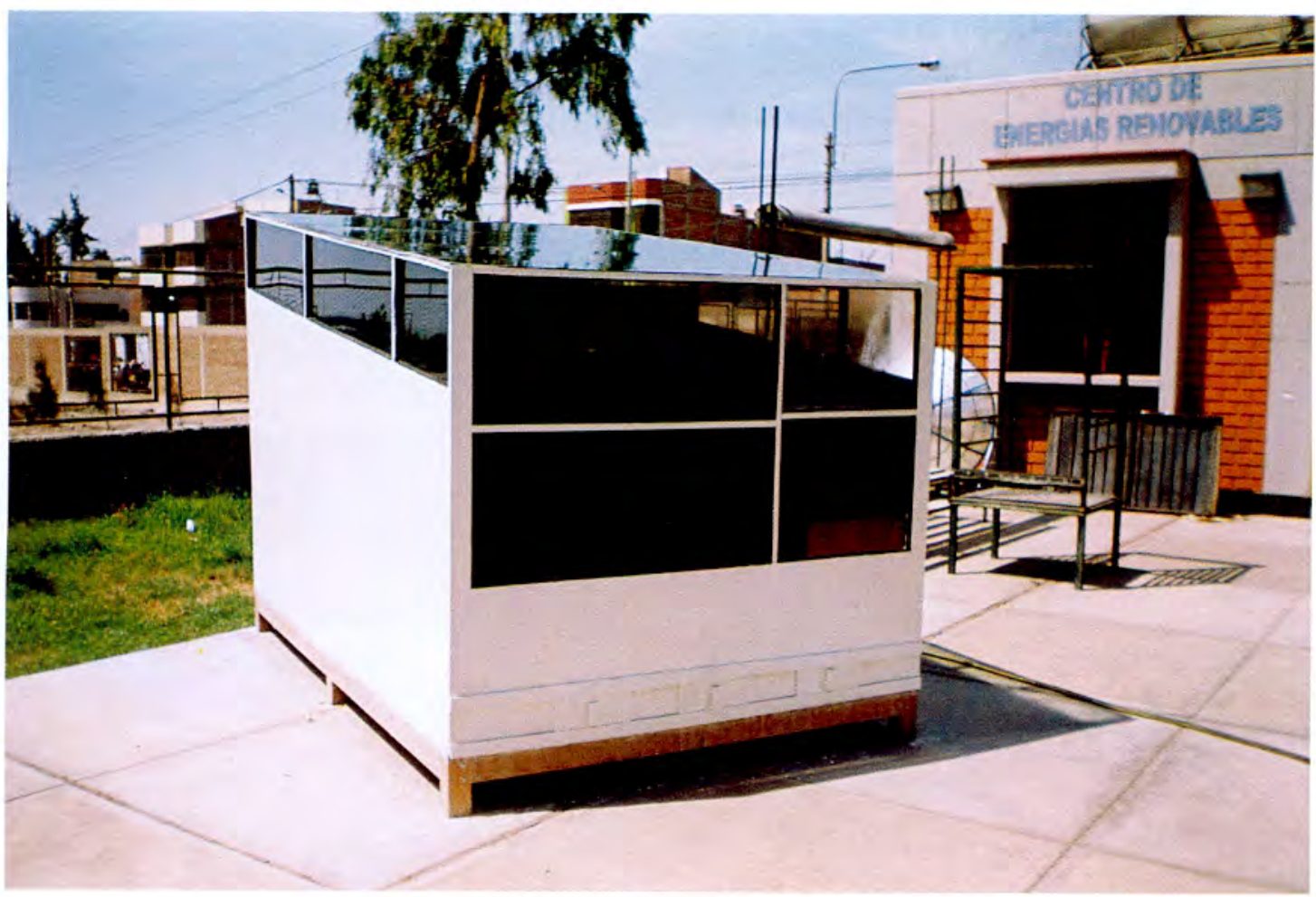

Figura $\mathrm{N}^{\circ} 03$. Vista frontal de la SSS

Fuente: Elaboración propia (2012) 
paredes, fondo, caja metálica - vidrio cobertor; vidrio cobertor - medio exterior; con las correspondientes ecuaciones, se determina que la eficiencia del sistema está dada por la ecuación siguiente:

$$
\eta=F \cdot \eta_{0}-F \cdot U_{P} \frac{\left(\bar{T}-T_{a}\right)}{G}-\frac{F \cdot m \cdot c}{A \cdot \Delta t}\left(\frac{T_{a c u m}-T_{a}}{G}\right)
$$

En la cual, $F$ es el factor de eficiencia con la cual se transfiere calor al aire interno, $\mathrm{h}_{0}$ es la eficiencia óptica del sistema, depende de la trasmitancia del vidrio cobertor, absorbancia de las superficies absorbentes, y del ángulo de incidencia de la irradiancia solar directa, $\mathrm{U}_{\mathrm{p}}$ el coeficiente global de pérdidas térmicas, T la temperatura promedio de aire interior, $\mathrm{T}$, la temperatura ambiente, $\mathrm{G}$ la irradiancia solar global incidente, $\mathrm{m}$ la masa térmica del sistema, calor específico promedio de todos los materiales utilizados para la construcción de la SSS, A es el área de captación de la irradiancia solar global, Dt el intervalo de tiempo, $\mathrm{T}_{\text {xum }}$ temperatura del acumulador térmico. Esta misma eficiencia del sistema la podemos estimar con la siguiente ecuación: (Acero Laura, G. 2012)

$$
\eta_{i}=\frac{m \cdot c \cdot\left(\bar{T}-T_{a m b}\right)}{\Delta t \cdot A \cdot G_{i}}
$$

\subsection{Radiación infra-roja}

Es la emitida por todos los cuerpos que se encuentran a una determinada temperatura, cuando ésta se incrementa la radiación emitida disminuye en longitud y aumenta su frecuencia, su aplicación es muy diversa, en el presente caso es de un ámbito medicinal y terapéutico (IRPA, 1985).

\subsection{Principio de funcionamiento}

El funcionamiento de la caja aislada térmicamente, está determinado por el efecto invernadero, el cual establece que cuando un recinto cerrado es expuesto a la irradiancia

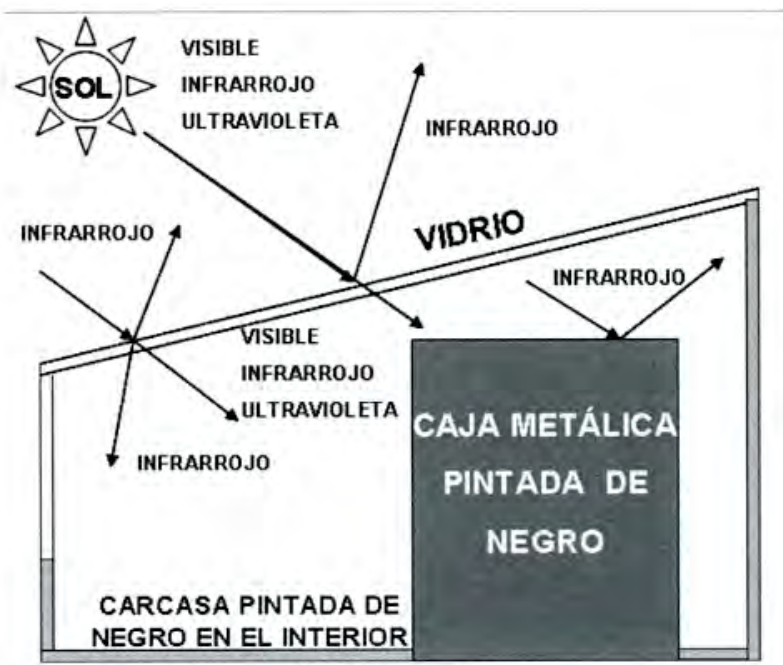

solar, la temperatura en su interior se elevará significativamente, si las propiedades ópticas de transmisión, reflexión y absorción de los materiales constituyentes son adecuadas. Para el caso de la SSS, el recinto cerrado y expuesto al sol, es precisamente la caja aislada térmicamente (Figura $\mathrm{N}^{\circ} 03$ ), cuya cubierta de vidrio deja pasar fácilmente casi toda la irradiancia solar incidente de longitud de onda corta, pero es totalmente reflector para la radiación larga emitida por el absorbedor, que al elevar su temperatura, emite radiación en la zona del infrarrojo, de este modo el calor radiante queda "atrapado" en la caja aislada térmicamente y las paredes del vidrio (Figura $\mathrm{N}^{\circ} 03$ derecha). (Acero Laura, G. 2011).

Las partes más importantes de la caja aislada térmicamente, tal como lo muestra la Figura $\mathrm{N}^{\circ} 04$-derecha, son la cubierta transparente, los absolvedores (caja metálica, paredes laterales y piso), el aislante térmico, la caja metálica pintada de negro, la Figura $\mathrm{N}^{\circ} 04$-izquierda es una vista de corte transversal de la SSS.

\section{MATERIALES E INSTRUMENTOS}

\section{Materiales}

Para la construcción y optimización de la SSS con acumuladores térmicos, se ha utilizado los siguientes materiales: 06 metros de madera de 1 pulg x $1 / 2$ pulg, 01 planchas de triplay de $4 \mathrm{~mm}$ de espesor, 02 planchas de vidrio de $1,5 \mathrm{~mm}$ de espesor, $03 \mathrm{~kg}$ de clavos de $21 / 2$ pulg y 1 pulg, pintura color negro mate, pintura de color blanco, 02 brochas, pegamento de madera, $619 \mathrm{~kg}$ de piedras de río, 214 litros de agua, 84 botellas descartables de 3 litros, herramientas de carpintería, herramientas de mecánica, material auxiliar (Acero Laura, G. 2011)

\section{Instrumentos}

Para la evaluación de la SSS construida se ha utilizado los siguientes instrumentos: 01 Piranómetro MollGorczynsky de la Kipp and Zonen, de sensibilidad espectral comprendido 0,3 y $3 \mathrm{~mm}$, con un tiempo de respuesta de 5 segundos y una constante de calibración de $10,35 \times 10^{-3}$

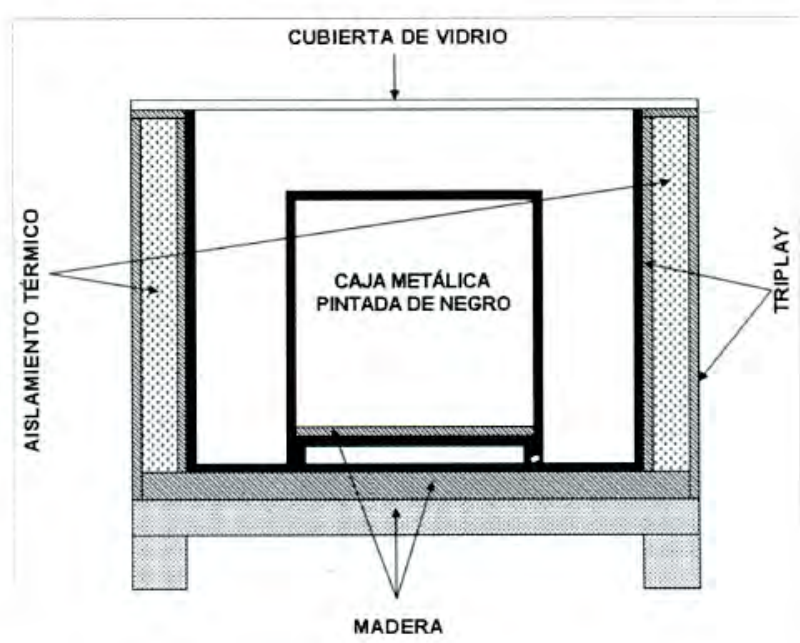

Figura $\mathrm{N}^{\circ}$ 04. Esquema del efecto invernadero de la SSS (derecha); vista de corte transversal de la SSS (izquierda) Fuente: Archivo propio (2012) 
$\mathrm{mV} / \mathrm{Wm} \mathrm{m}^{2}, 08$ termocuplas de Cromel - Alumel, como sensores de temperatura, ubicadas en diferentes partes de la sauna solar, 01 higrómetro digital Vaisala HM 34, 01 sistema de adquisición de datos marca Squirrel SQ 1200 series, 01 HOBO Weather Sttation H21-001, 01 cronómetro digital.

\section{RESULTADOS Y DISCUSIÓN}

\section{Resultados sin acumuladores térmicos:}

En la Figura $\mathrm{N}^{\circ} 05$, izquierda, se tiene una vista general de la SSS construida en el ambiente exterior del CERT, sin acumuladores térmicos, y en la derecha se muestra curva de la irradiancia solar global incidente sobe el plano del techo dela SSS en $\mathrm{W} / \mathrm{m}^{2}$ en función de las horas, para el día de la evaluación correspondiente; el gráfico de irradiancia nos indica que el día de la evaluación fue un día claro hasta las 15 horas, y en horas posteriores presenta nubes, alcanzando valores máximos por encima de los $900 \mathrm{~W} / \mathrm{m}^{2}$.

En la Figura $\mathrm{N}^{\circ} 06$, izquierda se muestra las curvas que definen el comportamiento térmico del proceso de calentamiento y enfriamiento diario de la SSS, bajo las condiciones de irradiancia solar global indicadas en la Figura
$\mathrm{N}^{\circ} 04$, específicamente temperaturas del sistema medidas en diferentes zonas: piso, parte media y parte superior de la SSS, así como la temperatura ambiente exterior. Puede observarse que la diferencia de temperaturas entre el ambiente exterior y la parte superior es mayor de $60{ }^{\circ} \mathrm{C}$, alcanzando valores máximos de $90^{\circ} \mathrm{C}$, para luego disminuir en la parte media y el piso, indicadores de que el sistema funciona, los valores máximos de la temperatura interna se alcanza alrededor de las 14 horas, para luego disminuir rápidamente en horas posteriores, en coincidencia con el perfil de la irradiancia solar global incidente; a partir de las 20 horas del día las temperaturas externas son casi iguales a la temperatura ambiente exterior.

La Figura $\mathrm{N}^{\circ} 06$, derecha muestra la potencia horaria disponible y útil ganada por la SSS, obtenidas considerando el área de captación del techo transparente de la SSS y la irradiancia solar incidente; en función de las horas del día, encontrando que la eficiencia media diaria de la SSS sin acumulador térmico, es de $38,7 \%$

Resultados con acumuladores térmicos de piedra sin pintar:
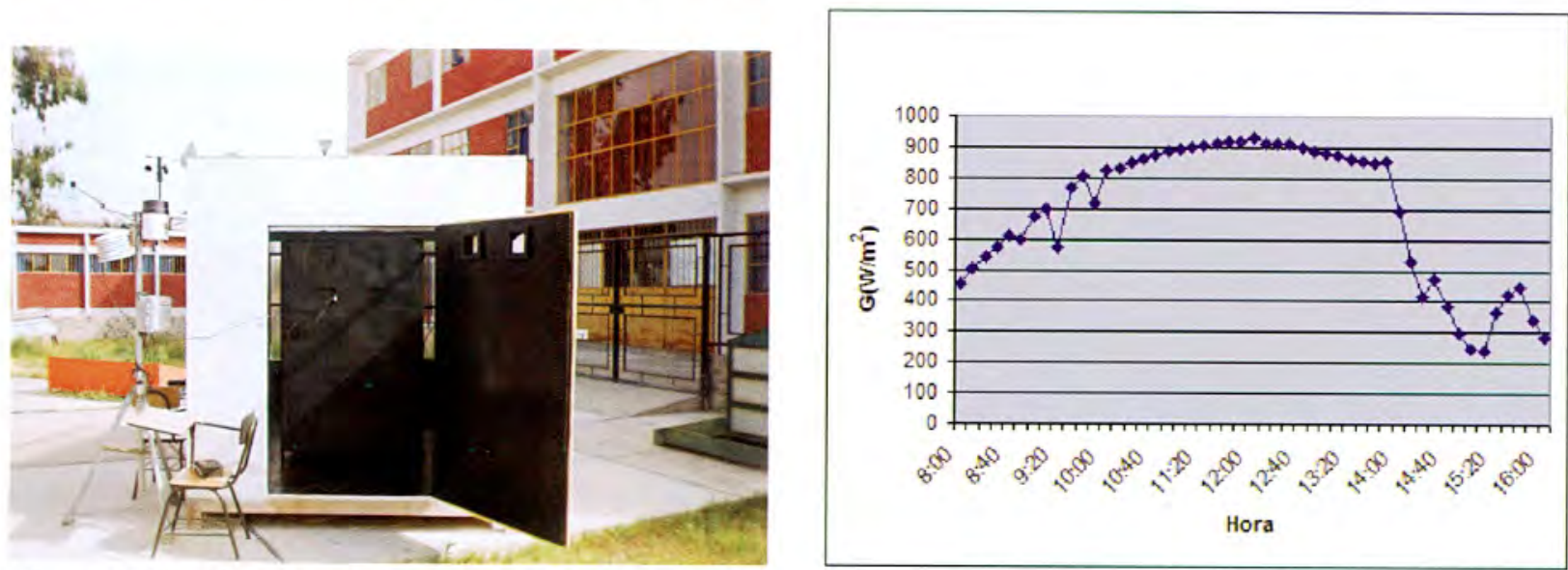

Figura $\mathrm{N}^{\circ} 05$. Vista general de la SS construido, sin acumuladores térmicos (derecha); irradiancia solar global incidente sobre la SS en función de las horas

Fuente: Archivo propio. (2013)
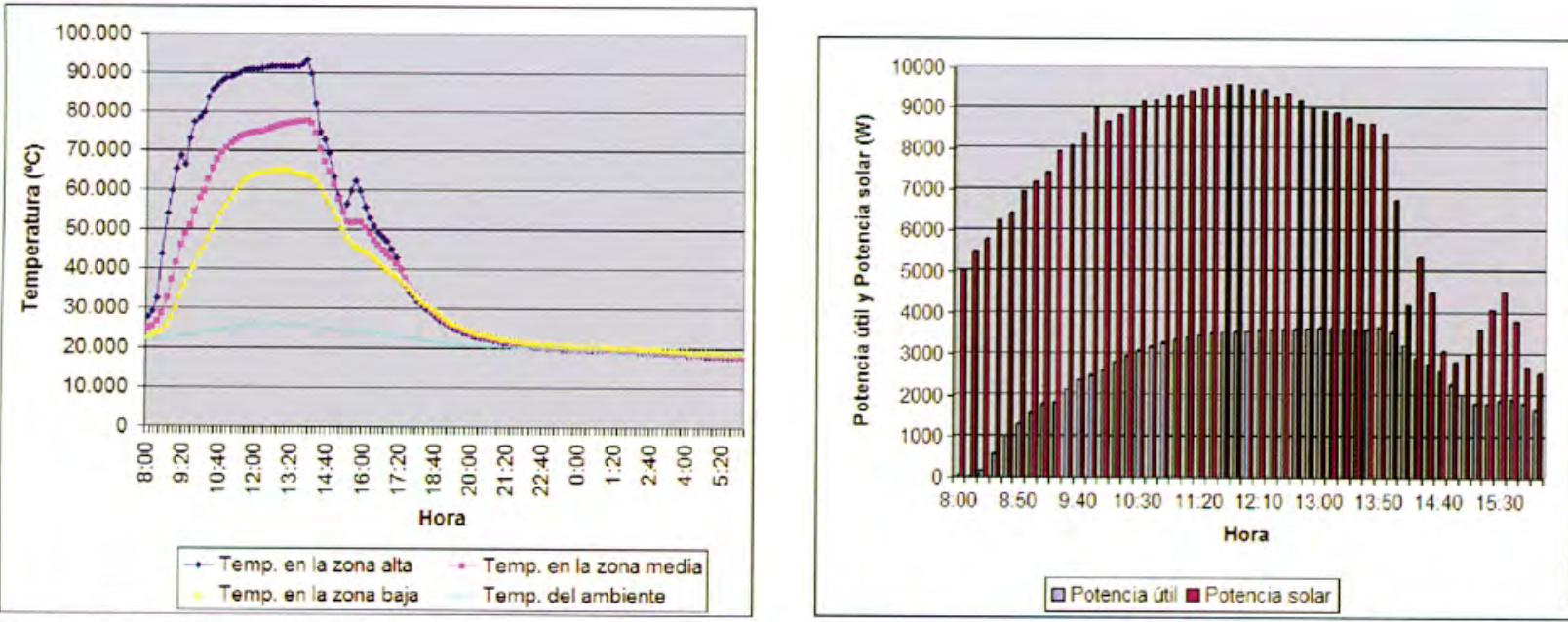

Figura $\mathrm{N}^{\circ} 06$. Curvas de perfil térmico para el calentamiento y enfriamiento de la SSS sin acumuladores térmicos en función de las horas del día (izquierda); Curvas de potencia útil y potencia solar disponible en función de las horas del día en la SSS (derecha)

Fuente: Archivo propio (2013) 
En este proceso se ha utilizado piedras de canto rodado de río con masa total de $619 \mathrm{~kg}$, con diámetro promedio de $15 \mathrm{~cm}$, con volumen total de $0,214 \mathrm{~m}$, colocadas en el piso de la SSS, tal como puede verse en la Figura $\mathrm{N}^{\circ} 06$, izquierda.

A efectos de poder comparar los resultados bajo las cuatro formas de evaluación de la SSS, sea elegido días claros, es decir cuasi completamente despejados, tal como se muestra en los gráficos de la Figura $\mathrm{N}^{\circ} 07$ y 8 derecha, en las curvas de perfil térmico del proceso de calentamiento puede observarse que el incremento de temperatura entre el ambiente exterior y la parte superior interna es de aproximadamente de $60{ }^{\circ} \mathrm{C}$, muy parecida al caso de funcionamiento sin uso de acumuladores, las piedras alcanzan temperaturas sobre los $70^{\circ} \mathrm{C}$, y durante las horas de enfriamiento la temperatura es mayor que cualesquiera de las otras partes del sistema; determinándose que las temperaturas máximas en el interior del SSS se mantienen hasta las 16 horas, casi cuatro horas adicionales respecto a sin acumuladores, asimismo la diferencia de temperaturas entre el interior y la ambiental se mantienen hasta casi las 03:00 horas, debido fundamentalmente al calor sensible acumulado por las piedras sin pintar, bajo estas condiciones la eficiencia media diaria de la SSS es de 38,09\%.
Resultados con acumuladores térmicos de piedra pintadas de negro:

En este proceso se han usado como acumuladores térmicos las piedras usadas en al caso anterior, pero pintadas integralmente con pinturas negro mate, con masa total de $619 \mathrm{~kg}$, volumen total $0,214 \mathrm{~m}^{3}$, y diámetro promedio de 15 $\mathrm{cm}$, colocadas en el piso de la SSS, tal como se visualiza en la Figura $\mathrm{N}^{\circ} 09$, izquierda. La curva 9 , derecha representa la irradiancia horaria solar global incidente sobre la SSS, la cual corresponde a un día completamente despejado a partir de las 09 horas, con valores máximos cercano a $900 \mathrm{~W} / \mathrm{m}^{2}$.

En la Figura $\mathrm{N}^{\circ} 10$, derecha se muestra el comportamiento térmico del sistema, usando como acumulador térmico las piedras del escenario anterior, pero pintadas con pintura negra mate, determinándose que las temperaturas en el interior de la SS en los tres niveles de medición alcanzan valores máximos superiores al caso anterior, el nivel superior pasa de $\operatorname{los} 94^{\circ} \mathrm{C}$, en tanto que las piedras alcanzan $\operatorname{los} 80^{\circ} \mathrm{C}$, como en el caso anterior durante las horas de enfriamiento mantiene temperatura superior al aire interno del sistema, la diferencia de temperatura entre el nivel superior y la ambiente externa es de aproximadamente $65^{\circ} \mathrm{C}$ durante las horas de irradiancia solar global máxima, mayor respecto al caso anterior ; bajo estas condiciones la eficiencia promedio diaria es de $40,7 \%$. Las temperaturas
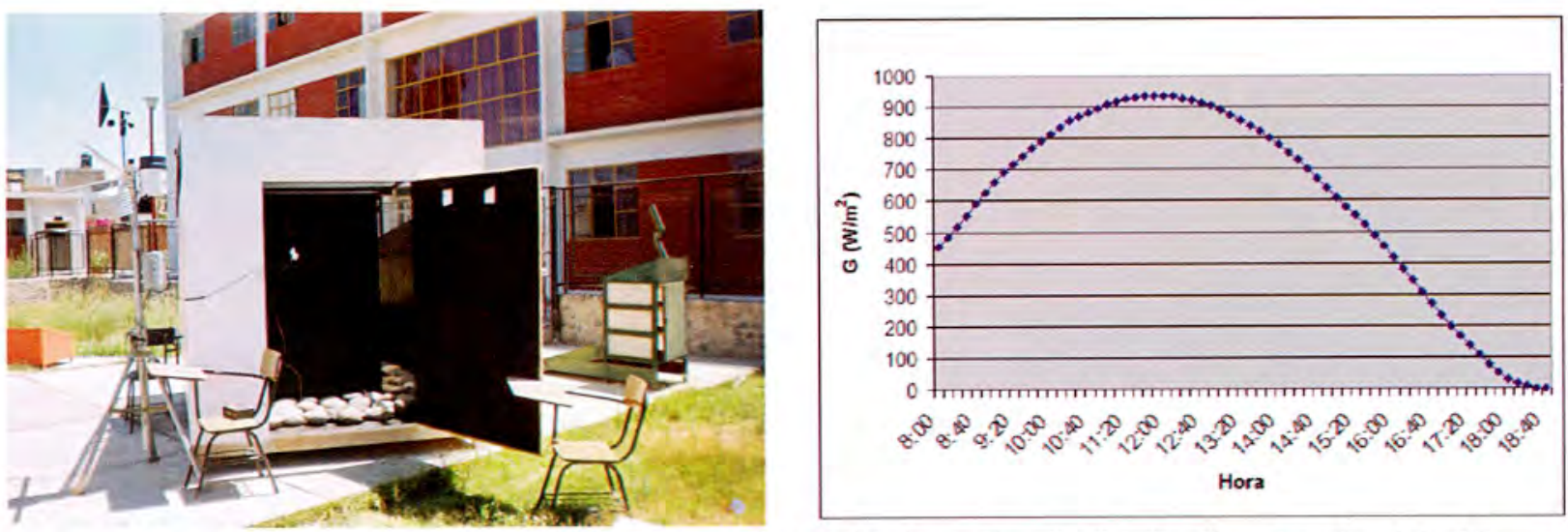

Figura $\mathrm{N}^{\circ} 07 . \mathrm{SSS}$ usando como acumulador térmico piedras sin pintar (izquierda), irradiancia solar global horaria incidente sobre la SSS (derecha)

Fuente: Archivo propio. (2013)
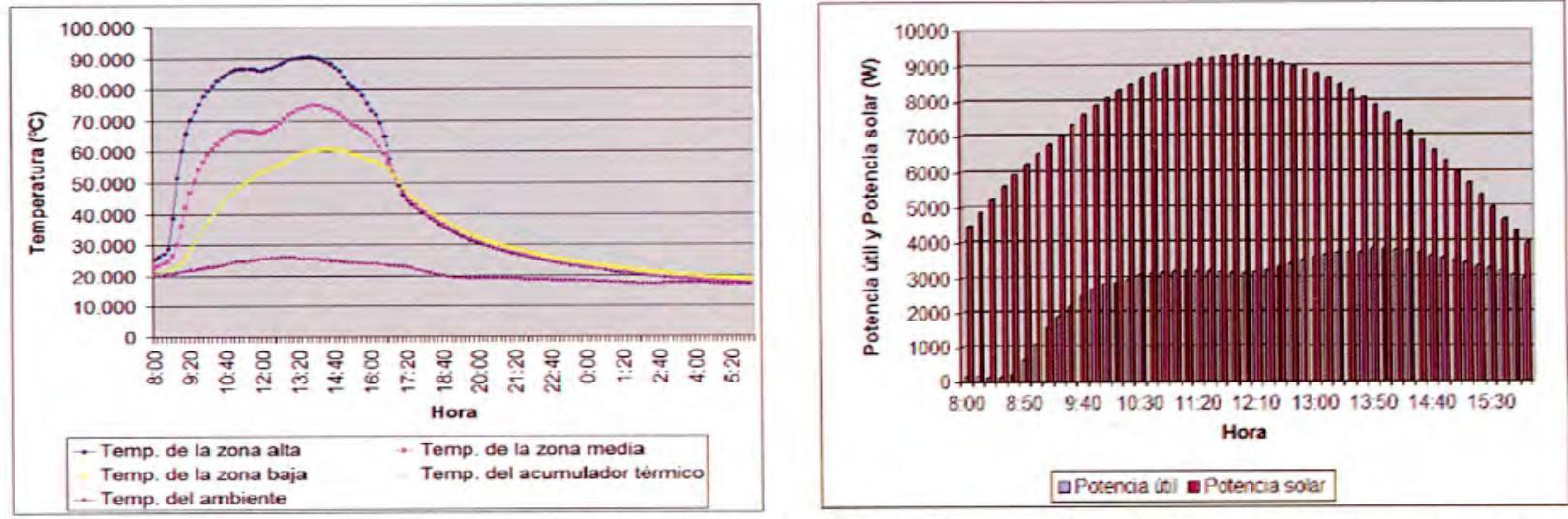

Figura $\mathrm{N}^{\circ} 08$. Curvas de calentamiento y enfriamiento de la SSS en función de las horas del día (derecha), Curvas de potencia útil y potencia solar disponible en función de las horas del día en la SSS (derecha)

Fuente: Archivo propio (2013) 


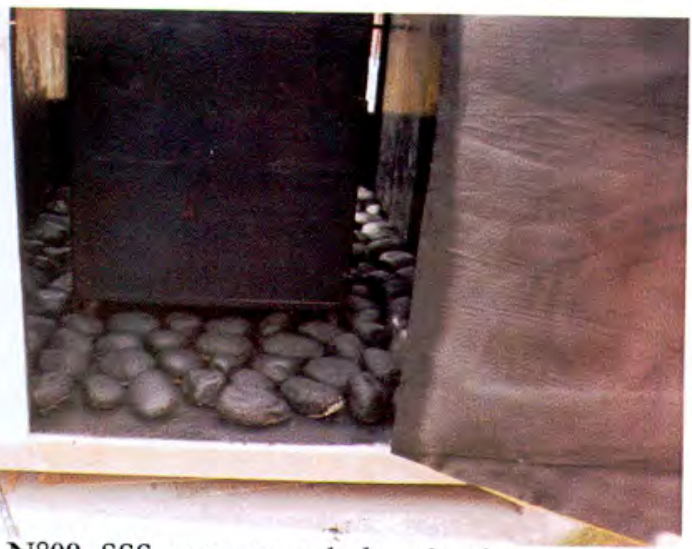

Bravo C. y Acero G., Caracterización y Optimización de una sauna solar seco con acumuladores témicos

Figura $\mathrm{N}^{\circ} 09$. SSS con acumulador térmico de piedras pintadas de negro mate, izquierda; curva de irradiancia solar global horaria incidente sobre la SSS, derecha.

Fuente: Archivo propio. (2013)
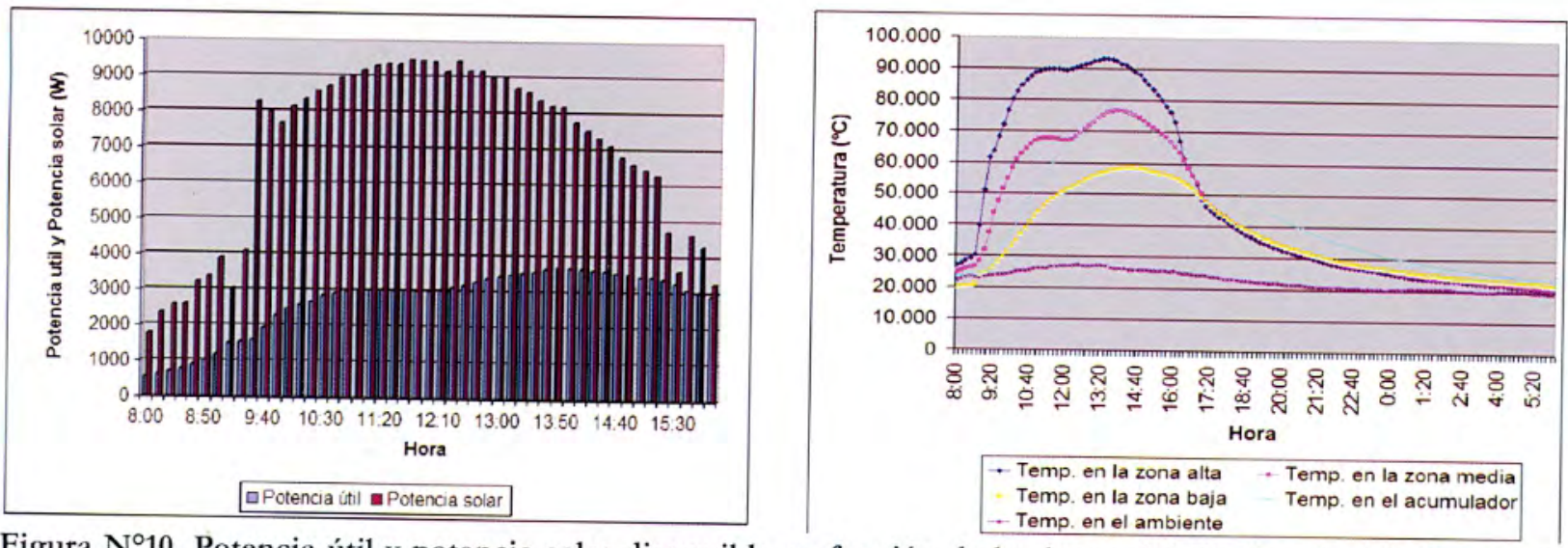

Figura $\mathrm{N}^{\circ} 10$. Potencia útil y potencia solar disponible en función de las horas del dia (izquierda), Curvas de calentamiento y enfriamiento en la caja metálica en función de las horas del día (derecha)

Fuente: Archivo propio (2013)

máximas en el interior se mantienen hasta las 16:00 horas, estas temperaturas se igualan a la ambiente exterior alrededor de las 03:00 horas.

Resultados con acumuladores térmicos de agua:

Para este escenario se han utilizado botellas de plástico transparente de gaseosa de 2,5 y 3 litros, llenas de agua potable, que hacen un volumen total de $0,214 \mathrm{~m}^{3}$, muy próximo al caso anterior a efectos de poder comparar el funcionamiento del sistema.

La Figura $N^{\circ} 11$ (derecha) muestra la irradiancia solar global horaria incidente sobre el sistema, correspondiente a un día semi nublado hasta las 10:00 horas, para el resto del día se presenta completamente despejado.

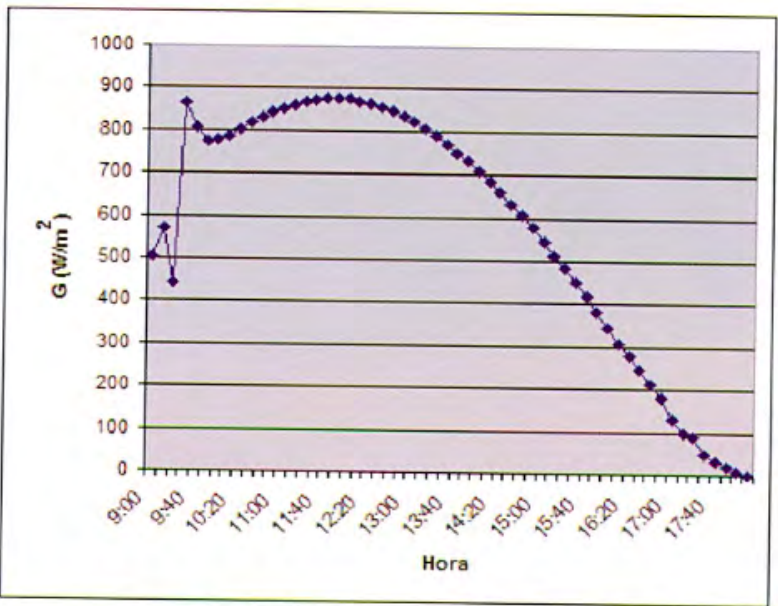

Figura $\mathrm{N}^{\circ} 11$. Vista de la SSS con acumulador térmico el agua contenida en botellas descartables (izquierda); curva de irradiancia solar global horaria incidente sobre el sistema (derecha)

Fuente: Archivo propio. (2013) 
La Figura $N^{\circ} 12$ (izquierda) muestra el perfil térmico para el proceso de calentamiento y enfriamiento de la SSS, usando como acumulador térmico agua contenida en botellas plásticas, la velocidad de calentamiento del sistema es mucho mayor que la de enfriamiento, los valores máximos de las temperaturas en el interior se mantienen constantes hasta las 16:00 horas, para luego disminuir a una velocidad lenta, en este escenario las temperaturas internas se mantienen por encima que la del ambiente exterior durante toda la noche. La diferencia de temperatura entre la parte superior del sistema y la ambiente exterior es de $63^{\circ} \mathrm{C}$; la temperatura máxima que alcanza el agua es de $58^{\circ} \mathrm{C}$, inferior a la alcanzada por las piedras; durante las horas de enfriamiento la temperatura del agua es superior que las otras partes del sistema, esto nos demuestra que las piedras y el agua actúan como fuente energética debido al calor sensible acumulado durante las horas de sol. Bajo esta condición la eficiencia media diaria es de $41,4 \%$.

\section{Resultados del comportamiento térmico-radiativo de} la caja metálica:

En la Figura $N^{\circ} 13$ (izquierda) el perfil térmico de la caja metálica pintada de negro para los cuatro escenarios de funcionamiento de la SSS, los valores de temperatura son valores promedios. Los valores máximos que alcanza es de aproximadamente $75^{\circ} \mathrm{C}$ entre las 11:00 y 15 horas, equivalentes a la de mayor irradiancia solar global incidente sobre el sistema, la velocidad de calentamiento es mayor que la de enfriamiento, con el uso de acumuladores térmicos la temperatura que alcanza es mayor, hasta las 20:00 horas del día, la diferencia de temperatura de la caja metálica y el ambiente exterior es superior a los $10^{\circ} \mathrm{C}$; la Figura $\mathrm{N}^{\circ} 12$ (derecha) muestra la longitud de onda de la radiación infrarroja horaria emitida por la caja metálica negra, la cual es función de la temperatura y calculada según la ecuación de Wien, variando entre 8 y 10 micras.

En la Tabla $\mathrm{N}^{\circ} 01$ se resumen los principales resultados de la evaluación de la SSS, específicamente las temperaturas máximas que se alcanzan bajo los cuatro escenarios de funcionamiento, el rango de la longitud de onda y la potencia emisiva de la radiación infrarroja emitida por la caja metálica pintada de negro, igualmente la eficiencia promedio del sistema

\section{Perfil de la humedad relativa:}

En la Figura $\mathrm{N}^{\circ} 14$ se observa el perfil de la variación horaria de la humedad relativa del aire interno de la SSS, en el interior de la caja metálica negra; así como el de la
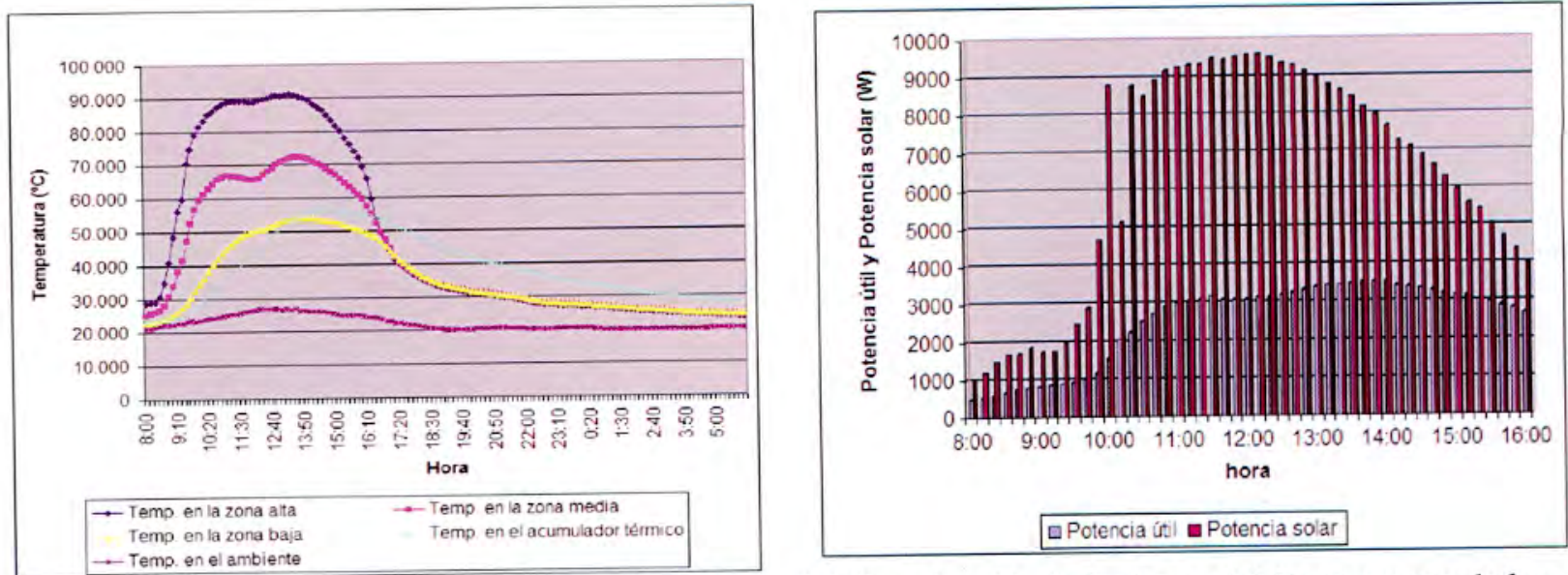

Figura $\mathrm{N}^{\circ} 12$. Curvas de calentamiento y enfriamiento en la SSS en función de las horas del día con acumuladores térmicos de agua en botellas (izquierda); Curva de potencia disponible y útil horarias en el sistema (derecha).

Fuente: Archivo propio. (2013)
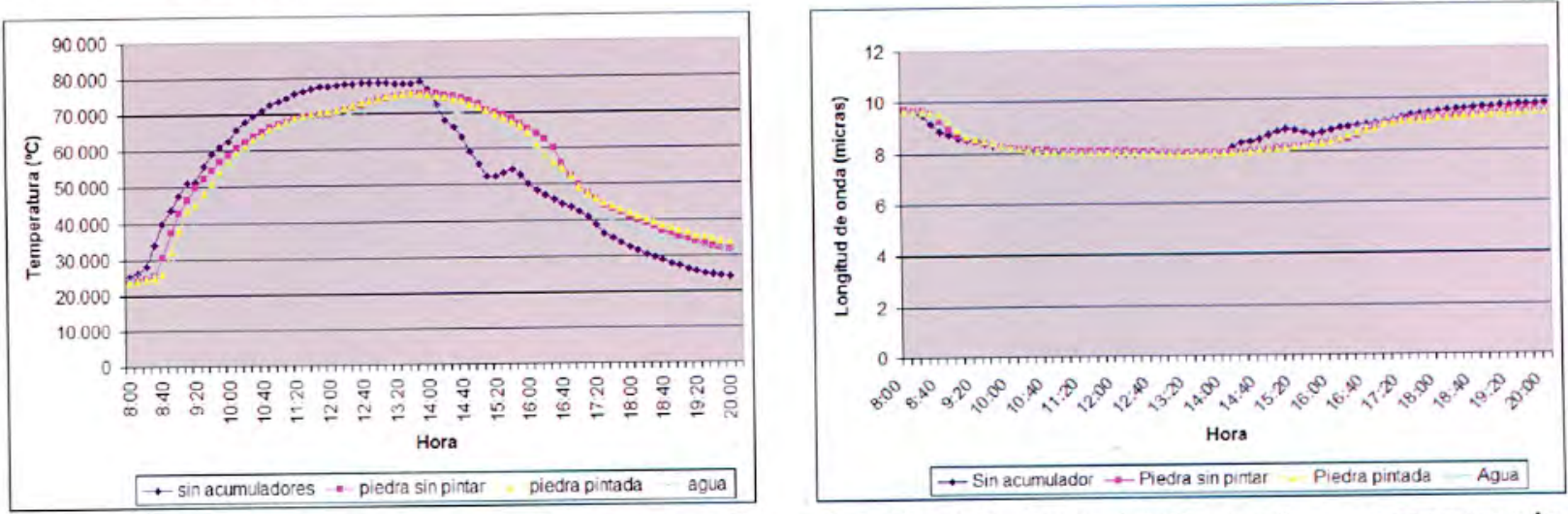

Figura $N^{\circ} 13$. Temperatura promedio de la caja metálica en función de las horas del día para los cuatro escenarios de funcionamiento (izquierda); Curva de radiación infrarroja horaria emitida por la caja metálica en los cuatro escenarios de funcionamiento (derecha).

Fuente: Archivo propio (2013) 
Tabla $\mathrm{N}^{\circ} 01$. Resultados experimentales de la SSS

\begin{tabular}{|c|c|c|c|c|}
\hline & $\begin{array}{l}\text { SSS } \sin \\
\text { acumuladores }\end{array}$ & $\begin{array}{l}\text { SSS con piedra } \\
\text { sin pintar }\end{array}$ & $\begin{array}{l}\text { SSS con piedra } \\
\text { pintada de } \\
\text { negro mate }\end{array}$ & $\begin{array}{c}\text { SSS con agua } \\
\text { contenida botellas } \\
\text { descartables }\end{array}$ \\
\hline Temperatura máxima en la SSS $\left[{ }^{\circ} \mathrm{C}\right]$ & 92 & 91 & 94 & 91 \\
\hline $\begin{array}{l}\text { Longitud de onda media mínima y } \\
\text { máxima emitida por la caja metálica } \\
\qquad[\mu \mathrm{m}]\end{array}$ & 9,8 a 7,9 & 9,55 a 7,97 & 9,5 a 7,9 & 9,5 a 8,0 \\
\hline $\begin{array}{l}\text { Potencia emisiva mínima y máxima } \\
\text { de la caja metálica }\left[\mathrm{W} / \mathrm{m}^{2}\right]\end{array}$ & 423,91 a 990,15 & 436,06 a 961,28 & 446,68 a 991,24 & 455,68 a 967,64 \\
\hline Eficiencia de la SSS $\eta_{d}[\%]$ & 38,7 & 38,1 & 40,7 & 41,4 \\
\hline
\end{tabular}

humedad relativa del aire ambiente exterior se determina que la humedad en el interior varía entre 30 al $8 \%$ durante el día, disminuyendo conforme se incrementa la temperatura interior, algo similar acurre con el contenido en el interior de la caja metálica, disminuyendo desde el $20 \%$ a inicios del día a $2 \%$ al final del mismo; mientras que la ambiental externa disminuye de un $70 \%$ a inicios del día, alcanzando valores mínimos de $48 \%$ entre las 12 a 15 horas, para luego incrementarse al $55 \%$.

\section{Análisis psicrométrico del aire interior de la SSS}

En la Figura $\mathrm{N}^{\circ} 15$ se muestra el comportamiento psicrométrico de la SSS, para el escenario del uso de acumulador térmico de piedras pintadas de negro mate, el punto "A" (líneas azules) representa el promedio de la temperatura ambiental de $24,08^{\circ} \mathrm{C}$, con la intersección con el de la humedad relativa ambiente promedio de $55,5 \%$, correspondiendo una entalpía de $52 \mathrm{~kJ} / \mathrm{Kg}$; el punto "B" (líneas rojas) representa el promedio de la temperatura del aire en la caja absorbente de $60,9^{\circ} \mathrm{C}$, el punto de intersección es con la curva de humedad relativa promedio $8,07 \%$ del aire en la caja metálica negra absorbente, correspondiendo una entalpía de $74 \mathrm{~kJ} / \mathrm{Kg}$, por lo tanto, la ganancia de entalpía del aire interno de la SS es de $22 \mathrm{~kJ} / \mathrm{kg}$ de aire.

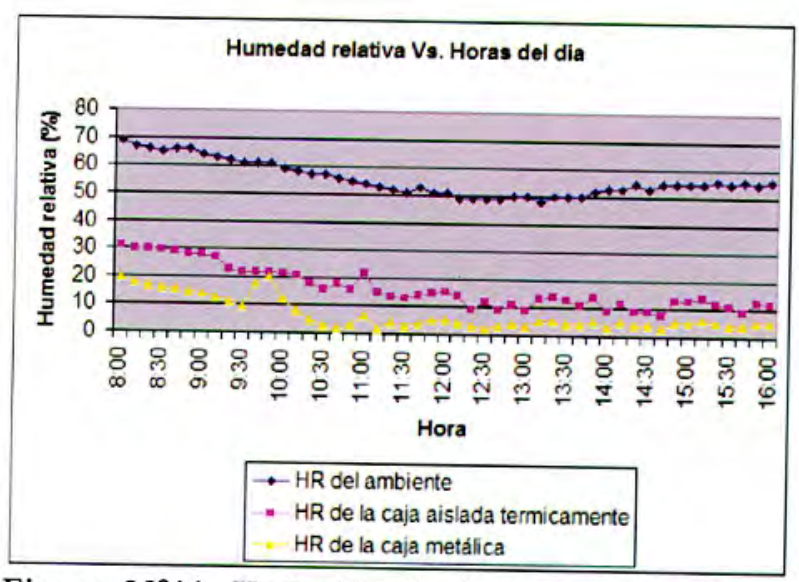

Figura $\mathrm{N}^{\circ} 14$. Variación de la humedad relativa ambiental, en el interior de la SSS y en la caja metálica negra, en función de las horas del día.

Fuente: Elaboración propia (2013)

\section{CONCLUSIONES}

Para los cuatro escenarios de evaluación, la velocidad de calentamiento es mucho mayor que la de enfriamiento, en la SSS con acumuladores térmicos la caída

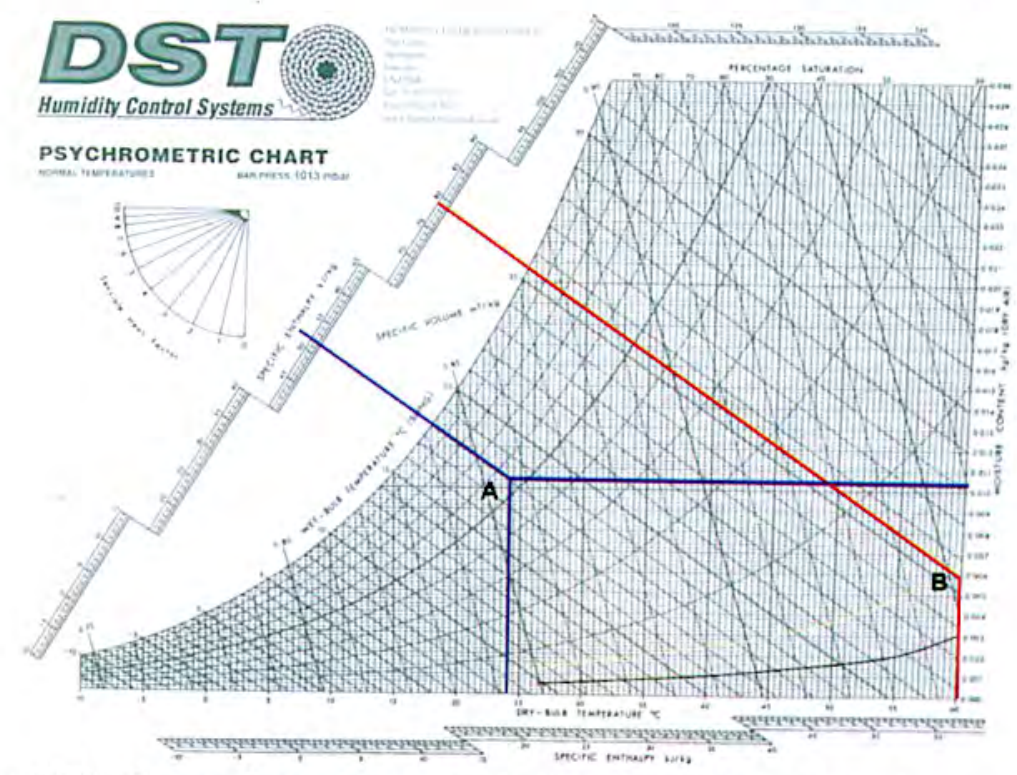

Figura $\mathrm{N}^{\circ} 15$. Carta psicrométrica para analizar la entalpía del aire interno de la SSS Fuente: Elaboración propia (2013) 
de temperatura ocurre entre las 14 y 15 horas, mientras que con acumuladores entre las $17 \mathrm{~h}$ y $18 \mathrm{~h}$, esto nos indica que el sistema se optimiza con el uso de acumuladores de piedra y agua.

Se determinó que la SSS alcanza la mejor eficiencia promedio con el uso de piedras pintadas de negro mate, equivalente a $41,4 \%$.

Con el uso de acumuladores térmicos en forma de calor sensible se obtiene que las temperaturas en el interior de la SSS se mantiene en promedio cuatro horas adicionales, respecto al uso $\sin$ acumuladores, manteniendo la temperatura interior del sistema por encima de la exterior ambiental hasta pasado las 03:00 horas del día siguiente.

Se determinó que, usando piedras pintadas de negro mate, se alcanza la mayor temperatura máxima en el interior de la caja metálica y de la SSS, igual a $94^{\circ} \mathrm{C}$, bajo irradiancia solar global incidente de $900 \mathrm{~W} / \mathrm{m} 2$.

Se determinó que la humedad relativa mínima en el ambiente interno de la SSS con acumuladores térmicos es de $1,6 \%$ en valor promedio, en tanto que la exterior ambiental en promedio diario es de $48 \%$.

La caja metálica pintada de negro actúa como una buena fuente emisora de radiación infrarroja con longitudes de onda en el rango de 8 a 10 micras la cual depende de su temperatura; con una potencia emisiva que oscila entre $423,91 \mathrm{~W} / \mathrm{m} 2$ a $991,24 \mathrm{~W} / \mathrm{m} 2$ durante las 08 a 20 horas, este resultado sugiere que la integración entre la SSS y la cabina de radiación infra-roja es viable para aplicaciones terapéuticas.

\section{RECOMENDACIONES}

Para el diseño del área de recepción de la radiación solar se debe tener en cuenta, que el ancho sea mayor que el largo, según el desplazamiento del sol de este a oeste, para lograr mayores temperaturas durante todo el día.

No usar materiales conductores del calor en la estructura interna de la SSS para disminuir las pérdidas de calor. Localizar todas las posibles fugas de calor, para mejorar el aprovechamiento del efecto invernadero.

En el diseño, tener en cuenta las condiciones meteorológicas del lugar, como: lluvia, humedad, polvo, nieve, etc., a efectos de evitar fugas y filtraciones que afectan la vida útil del sistema

\section{REFERENCIAS BIBLIOGRÁFICAS}

Acero Laura Gohnny, 2012, Diseño, Construcción J Caracterización de una Sauna Solar Seco, Facultad de Ciencias, ESFI Universidad Nacional Jorge Basadre Grohmann, Practicas Pre-Profesionales.

Acero Laura Gohnny, 2013, Optimización y Caracterización de una Sauna Solar Seco con acumuladores térmicos, Facultad de Ciencias, ESFI, Universidad Nacional Jorge Basadre Grohmann, Tesis de titulación

Asociación Internacional para la Protección contra las Radiaciones (IRPA), 1985, Guidelines for limits of buman exposure to laser radiation, Health Pbys 48(2), 341-359.

Chassériaux J.M., 1990, Conversión térmica de la radiación solar, Librería agropecuaria S.A., primera edición, Buenos Aires -Argentina.

Duffie John y Beckman William, 1991, Solar Engineering of Termal Processes, Editorial John Wiley y Sons, INC, Segunda edición, EE.UU

Yunus A. Cengel, Jhon M. Cimbela, Robert H. Turner (2012); Fundamentals of termal-Fluid Sciences; cuarta edición, Mc Graw Hill, USA

Fernández Diez Pedro, 2005, Procesos Termosolares en baja, media y alta temperatura, Universidad de Cantabria, Primera edición, España.

Ordóñez Miranda Edilberto José, 2004, Construcción y Evaluación de una Terma Solar Tipo Termosifón de Colector de Placa Plana con Rejillas de Cobre, PVC y CPVC, Universidad Nacional Agraria La Molina, ESFI - Universidad Nacional Jorge Basadre Grohmann, Tesis de titulación

\section{Correspondencia:}

Carlos Polo Bravo: polobravocarlos@yahoo.es

Gohnny Acero Laura: yhonacer@gmail.com
Fecha de Recepción: 23/06/2014

Fecha de Aceptación: 02/07/2014 